\title{
Jeder Mensch hat Christus in sich*
}

B. Staehelin

\section{Christus als das neu Modernste}

Wie komme ich dazu, als Mediziner, als Psychotherapeut über Gott, über Gottes Sohn und über Gott Heiliger Geist öffentlich zu sprechen - theologisch gemäss der Theologie unserer Landeskirchen?

Dazu noch: ich weiss, dass ich ein Sünder bin, war und wohl immer bleiben werde. Ich sollte also bei religiösen Dingen besser schweigen oder warten, bis ich vollkommen sein werde - wie wohl der Sohn Gottes ausgesprochen gern zu den Sündern gekommen ist.

Schwiegen wir Sünder aber immer und suchten nicht, über Gottes Wahrheiten zu sprechen und Gottes Stimme in unserem Innern und im Innern des Nächsten, der Nächsten im Gebet zu vernehmen, dann änderte sich in einer heute mehr noch gottlosen Psychotherapie, Medizin, Wissenschaft, Politik, Gesellschaft, Alltag nichts, nichts auf Gott hin.

Und nun aber gerade in diesen Jahren geht der 400jährige gottlose Zeitgeist der Aufklärung und der Entheiligung, Säkularisation wieder über in einen Gott und seine Wahrheiten neu suchenden neuen Zeitgeist.

\section{Die christliche Therapiearbeit ist am Beginn einer neuen Glaubensepoche}

Die Schulmedizin, die Spitäler und die Arztpraxen in den USA und England, in zunehmender Weise auch in der deutschen Schulmedizin, sind in einer schon grossartigen Art offen und praktizierend für Christus, offen für Gebet, christliches Beten, Fürbitten, christliches Gebetsheilen, durch Liebegeben. In der Schweiz sind wir erst am Erwachen für diese neuen Gebetstherapien. Wir sind erst beim erwachenden Augenreiben. Aber manche kämpfen schon für den Einzug des oft helfenden und heilenden Christus medicus in die Schulmedizin.

Ich selbst habe eine Gebetstherapie entVortrag - stark gekürzte Fassung gehalten am 2. Februar 2002 in Zürich an einer Psychotherapieveranstaltung von Dr. Martha von Jesensky.

Korrespondenz:

Prof. Dr. med. Balthasar Staehelin Freiestrasse 108

CH-8032 Zürich 7 wickelt - die psychosomatische Basistherapie auch Christustherapie genannt - und führe seit Jahren eine entsprechende Gruppenarbeit. Auch sind wir daran, eine schweizerische Verbindung für christliche Psychotherapie aus der Taufe zu heben.
Unsere mitarbeitende Frau Katharina Bär möchte ich hier darum erwähnen, weil sie die fähige, geeignete Organisatorin, die Verlässlichkeit ist für diese unsere neuen Aktivitäten.

\section{Vom psychosomatischen, vegetativen Psychosyndrom}

Das häufigste Störungsbild in der gesamten Medizin ist das psychosomatische, vegetative Allgemeinsyndrom, ist das Leib-Seele-Gemeinschaftsgeschehen. Etwa ein Drittel aller Patientinnen und Patienten in den Sprechzimmern der Allgemeinärztinnen und -ärzte leidet darunter.

Ich habe auf der medizinischen Poliklinik des UniversitätsSpitals Zürich während 35 Jahren die psychosomatische Sprechstunde geführt. Ich habe wissenschaftlich vornehmlich mit diesem psychosomatischen Angstsyndrom gearbeitet und tue das auch heute weiter in meiner Privatpraxis. 35 Jahre lang habe ich diese angstvollen Patientinnen und Patienten vor allem nach der Ursache und der Behandlung untersucht und dabei auch eine spezielle Therapieform entwickelt: die psychosomatische Basistherapie eine Therapieform, die den Glauben an den dreifaltigen Gott aufbauen, vergrössern und vertiefen will.

Das in die Augen springende Wesentliche für die Therapie sehe ich darin, dass diese psychovegetativen Patientinnen und Patienten in ihrer Grundstimmung zuwenig Vertrauen, zuwenig Urvertrauen, zuwenig Halt bekommen haben, zuwenig Glauben, Hoffnung, zuwenig Liebe.

Das naturwissenschaftliche Menschenverständnis als die Grundlage der derzeitigen Schulmedizin kennt den Menschen, seine Psyche und sein Soma, seine Psychosomatik, seine charakteristische Unsterblichkeit nicht. Vor der Unvergänglichkeit jedes Menschen Natur steht die nur naturwissenschaftliche Schulmedizin noch etwas verständnislos gegenüber.

Die Unvergänglichkeit jeder Natur des Men- schen verlangt deshalb für ihr Morgen ein Menschenbild, das in der ewigen Eigenschaft des göttlichen Haltgebens, des Liebegebens und des ewigen Vertrauengebens seine therapeutischen Wurzeln hat. Ich fand und finde dieses entscheidende Menschenbild im christlichen mystischen Menschenverständnis. 


\section{Jeder Mensch hat Christus in sich}

Viele Menschen, unsere Mitmenschen, wir, mit oder ohne vegetativem Allgemeinsyndrom - wir haben es soeben angedeutet - haben Angst, unbewusst, haben zuwenig inneren Halt, zuwenig Glauben, zuwenig Liebe. Sie leben nicht vom zwar meist unbewussten Gefühl, von etwas Ewigem Heiligem gehalten, geführt und ernstgenommen $\mathrm{zu}$ werden. Sie haben nicht das Empfinden, wie es der seelisch ganz Gesunde hat: es wird schon irgendwie (in alle Ewigkeit) gut gehen.

Diese verängstigte, neurotische Haltlosigkeit, diese Glaubensunfähigkeit, dieses Fehlen von genügendem Urvertrauen, Weltvertrauen, dieses Mangelhafte ist nun in der Therapie dieser so häufigen Krankheiten das zentrale Anliegen. Die Fähigkeit des Menschen, glauben zu können, dass alles schon einmal gut kommen wird - diese Grundstimmung zu erreichen, ist das Ziel solcher Behandlung. Es bieten sich hier an: Schulmedizin, Komplementärmedizin, Physiotherapie, Medikamententherapie, kleine und grosse Psychotherapie, christliches Gebetheilen.

Und nun, das was am meisten Urvertrauen, Selbstvertrauen, Weltvertrauen, Gottvertrauen, Christusvertrauen herbeibringen kann, ist dasjenige, was in den vier Evangelien des Neuen Testamentes der Bibel - am auffälligsten im Johannes-Evangelium beschrieben, versprochen ist. Der dreifaltige Gott, Gott Vater, Gott Sohn, Gott heiliger Liebesgeist, bieten immerwährenden Halt, Boden, Vertrauen, Geborgenheit, Heimat, Ewigkeitsein an, bieten an des Menschen Fähigkeit, an Gott zu glauben, an Jesus Christus glauben zu können.

Und auch dieses hat sich mir im täglichen Umgang mit Starken, Gesunden und mit den Patientinnen und Patienten mit dem psychosomatischen, vegetativen Psychosyndrom sowohl durch verstandesmässige Beobachtung als auch durch herzensmässige Erfahrung und durch Glaubensgewissheit ergeben: Christus ist in jedem Menschen.

Das ist auch der Grund, warum ich in meiner psychotherapeutischen Praxis immer mehr diesen uns so nah anwesenden Christus mit Gebet und Gespräch - für dafür geeignete Kranke bitte, an der Therapie mitzumachen. Ich möchte eine neue, christliche Gebetspsychotherapie aufbauen.

Das Gottesbild und das Menschenbild des Johannes-Evangeliums lassen sich dahin zusammenfassen: aus dem unsichtbaren Gottvater im Himmel und aus dem unsichtbaren Gottsohn, der überall ist, fliesst immer aus, geht immer hervor und überallhin, auch in jeden Menschen.
Und dieser Liebesgeist Gottes zeugt, lässt im Glaubensorgan jedes Menschen guten Willens hervorgehen den Menschensohn, Christus.

In diesem Marienorgan - wie ich sage - im Glaubensorgan jedes Menschen geht hervor in des Menschen auferstehende Bewusstheit der tatsächliche Sohn Gottes mit seinen strahlenden Eigenschaften.

Dieses fundamental wichtige Ereignis trägt in der christlichen Theologie den Namen: die immerwährende Kommunion - das immerwährende Abendmahl. Ich schlage auch diese Bezeichnung vor: «mystischer Liebesgeist zwischen Gott und dem Menschen». Wäre das Wirken der christlichen Dreifaltigkeit nicht dieser Art, es gäbe keinen Menschen. Denn das Leben in jedem Menschen ist der dreifaltige Schöpfergott Christus. Die immerwährende Kommunion - das sich Ergiessen des Geistes Christi immerwährend Augenblick für Augenblick - in und um jeden Menschen, ist das wichtigste Geschehen für jeden Menschen. Es ist Weg, Wahrheit, Leben, Heilsweg, Heiligungsweg, ewiges Leben.

Ohne dieses Einwirken Gottes in die Inwendigkeit jedes Menschen gäbe es kein vergängliches und kein unvergängliches Leben für den Menschen.

Wenn es aber Wahrheit ist, dass das naturwissenschaftliche Menschenbild sich ergänzen muss mit diesem spirituellen, mystischen Menschenbild, dann hätten wir auch in der Psychotherapie, in der Psychiatrie, der Psychosomatik und Psychologie diesen Christus in jedem Menschen aufzunehmen in diese Fächer.

\section{Fallbeispiel einer Gotteserfahrung}

Mir scheint es angebracht, hier ein Fallbeispiel einer christlichen Gotteserfahrung anzugliedern.

Vor etwa vier Jahren behandelte ich psychotherapeutisch eine damals 45jährige Geschäftsfrau. Die Frau litt an einem starken psychovegetativen Syndrom mit angstvoller Grundstimmung, reaktiven Depressionen und an einer Neigung zu Magen-Darm-Störungen. Sie hatte ein grosses Sehnen nach Gott, ohne aber schon einen gefestigten, christlichen Glauben $\mathrm{zu}$ haben. Die Behandlung konnte mit Erfolg abgeschlossen werden - es war eine Gesprächstherapie, einmal wöchentlich, ohne Medikamente. Der christliche Glaube kam sehr oft zur Sprache. Vor einigen Wochen erhielt ich einen Brief von dieser ehemaligen Patientin: sie teilte mir mit, dass sich ihr durch regelmässiges, tägliches Beten eine Gotteserfahrung eingestellt hatte. Für diese Gnade ist sie sehr dankbar. Sie hatte ihre diesbe- 
zügliche gnadenvolle Erfahrung schriftlich festgehalten. Die Niederschrift dieser Erfahrung schenkte sie mir. Ich darf frei darüber verfügen. Diesen Brief hier lasse ich ohne Kommentar für sich sprechen. Nur soviel: wir haben hier eine selten klare, überzeugende und schlichte Darstellung darüber, was eine echte Gotteserfahrung sein kann. Die Frau hatte in den letzten drei Wochen den hier erwähnten Seligkeitszustand während einigen Tagen und Nächten.

\section{«Lieber Herr Doktor}

Mein Herz jubelt, denn Christus ist immer anwesend. Er ist jede Sekunde anwesend, und sein grösster Wunsch ist es, dass wir Menschen dies glauben und spüren können. Wir sind ununterbrochen von seiner grossen Liebe berührt. Ja, er möchte das Beste für jeden Einzelnen von uns. Wir können nie ins Leere fallen, denn seine Hände halten uns auf sicherem Boden. Was auch alles Schlimme geschehen mag, er lässt uns nie im Stich! Er liebt uns unermesslich, und seine Liebe ist ohne Anfang und ohne Ende. Wir sind umhüllt von seinem Heiligen Geist: Christus stärke unseren Glauben, damit wir fähig werden, Deine Grösse in jeder unserer Zellen zu vermehren. Nichts kann uns anhaben, denn Du bist der Beschützer, Tröster, der Grossgeliebte in uns. In der weltlichen Liebe schenkst Du uns einen Menschen, bei dem wir ansatzweise Deine grosse Liebe erfahren dürfen. Wir danken Dir für Deine Geduld mit uns. Du weißt, zu was allem wir fähig sind ohne Deine Gegenwart. Du schenkst uns das Grösste, Beste, und wir sind nicht fähig, all Deine Wunder in ihrer Grösse zu verstehen. Schenk uns Weisheit und Geduld für alles, was uns der Alltag zuträgt. Wir danken dir, Jesus
Christus, für Deine Güte und Liebe. Mach uns fähig, Dir immer ähnlicher zu werden. Die Welt benötigt viele Menschen, die Deine Liebe an den Nächsten weitergeben, Zuversicht und Licht ins Dunkle tragen zu können. Deine Liebe, mein Jesus, mein Geliebter, will ich stets tief in meinem Herzen bewahren und weitertragen. Vergib uns unsere Verfehlungen, Sünden, die wir so oft unbewusst machen und nicht erkennen und unterscheiden können, ob es Dein Wille ist oder der Satan uns ins Ohr einflüstert. Alles geschehe nach Deinem Willen!»

\section{Zusammenfassung}

Lass uns glauben, dass das, was Dir Jesus Christus tut, immer im Grunde das Beste und das Schönste ist - entsprechend dieser Psychotherapieempfehlung: sich soviel als möglich mit dem Besten von allem Guten und mit dem Schönsten von allem Schönen beschäftigen: dieses Beste und dieses Schönste ist der Sohn Gottes, Jesus Christ in Dir. Dieser Weg führt in die Gesundheit - und dann in das Ewige Leben, bei und mit Gott.

\section{Literatur}

Die hier verwendete Literatur ist aufgelistet in Staehelin B. Mensch und Menschensohn. 3. Auflage. Udligenswil: Assisi-Verlag; 1996, ISBN 3-9520193-01-9. Ab 1. Oktober 2002 erscheint im Herder Verlag, D-Freiburg im Breisgau, das neue Buch von Balthasar Staehelin: Heilung geschieht von innen, mit weiteren Literaturangaben. 